\title{
Mobile Peer-to-Peer Technology Used in Mobile Education Field
}

\author{
Liang Ye and Ci Lin-lin \\ School of Computer Science and Engineering, Beijing Institute of Technology, Beijing \\ 100081, China \\ Liang-ye@sohu.com, cilinlin@263.net
}

\begin{abstract}
Mobile Education is a new way of using wireless and mobile technologies for education, and it usually applies to nature-based field study. However, the fee of use is so high that few people could accept this new learning method. But we take note of that the message, required by users coming from the same group with wireless communication equipments, usually focuses on a certain area. So, we could use the mobile peer-to-peer (MP2P) technology to resolve this confliction. MP2P network can let messages be transferred in the group instead of in the whole communication system, and this could save the messages in the group and meet the need of other users through storage and transmitting. So the users could cut down the fee of using mobile education system.
\end{abstract}

Keywords: Mobile P2P, Mobile Education, Wireless Communication.

\section{Introduction}

Mobile Education, or M-Education, is a new way of using wireless and mobile technologies for education by extending access to a desktop-based online virtual environment called MOOsburg [1] to handheld devices used as part of a mobile collaborative community. That means, we can get knowledge all over the time, and be not limited by where we are. In mobile education field, the handheld mobile devices, i.e. mobile phone, could help us to get information. At the same time, numerous schools begin to build resource servers and provide education resources for their students, especially the resources which may be used in the field study. When the students take part in a nature-based field study, for example, the geology field course, they usually come to be perplexed by a lot of nature phenomena. And they also want to share their discovery with their classmates, so that they could collaborate with each other. In this case, they need use the phones to download or upload the message with the fixed server in the school.

But we know that, the fee of using those mobile equipments is very high, so we couldn't use them for all things we want, and this may affect the effect of this geology field course. In order to reduce the fee for communication, the mobile peer-to-peer (MP2P) technology is proposed. Using MP2P technique, we can reduce the rate of communication. That is, the fee for communication is low, and we can use mobile devices for study on a larger scale. 
This paper is organized as follows. In Section 2, we briefly summarize related work. In Section 3 we present the technique of organizing a MP2P network. Comparing results and conclusions are reported in Section 4.

\section{Related Work}

Numerous efforts are being made in the direction of using handheld mobile devices for the purposes of sharing educational resource. Some technologies simply store the shared educational resources in the database server, and distribute these resources through browser / server $(\mathrm{B} / \mathrm{S})$ mode or client / server $(\mathrm{C} / \mathrm{S})$ mode to users' handheld devices. Other technologies plan to realize the function of sharing resources by means of the third generation mobile phone. By examining a few related applications and concepts, we shall see how MP2P makes mobile education technologies using wireless and mobile devices one step further.

Now, most people get information using the traditional network technique, which base on $\mathrm{B} / \mathrm{S}$ or $\mathrm{C} / \mathrm{S}$ network mode. But there are two disadvantages to this technique: On the one hand, the bandwidth of wireless link is a major performance bottleneck. The more users want to get the similar resource from server at the same time, the more response time is needed for each user. On the other hand, wireless communication is very expensive, and the burden of fee would fell to the users after they use this kind of application for a long time.

There are some technologies designed for the third general mobile phone. These technologies mainly concentrate on how to use P2P technique in the mobile communication field. But yet, P2P technique can't be used on the mobile phone of $2.5 \mathrm{G}$ networks generation, in which an IP address, unlike the third generation mobile phone, can't be distributed for each, the research on $\mathrm{P} 2 \mathrm{P}$ for mobile phone only has theoretic value.

In the interest of the users, we propose a new technique, MP2P technique, for mobile education. Through the MP2P technique, we can get the education resource we need with shorter response time and lower fee via our mobile phone.

\section{Organizing a MP2P Network}

In mobile environment, handheld mobile devices are always moving; signal might break off frequently; the bandwidth of wireless link is narrow 2]. As a result, we need to design a new network organizing technique to suit for it. It consists of group management technique, resource management technique, and pipe technique.

\subsection{Group Management Technique}

Group management technique includes creating, entering, and exiting group.

At first, all the handheld mobile devices use Bluetooth module or Ad hoc module to connect with each other and buildup a local MP2P network, and all 
of them makeup a basic group. When a task, for instance, downloading a map all about them, is brought forward in the basic group, a device which could do it immediately is selected to buildup a new sub-group by the mobile agent running in the MP2P network, and this device is called leader of the new group.

After the leader device builds up a new group, the devices which maybe use the same resource join into the group one by one. But how to decide whether a device needs to enter the group? In order to explain the regulation in detail, we put forward some definitions.

Definition 1 Relativity: Suppose there are two devices named $N_{A}$ and $N_{B}$ respectively, the aggregate of attributes for $N_{A}$ is $A_{\text {attribute }}$, and for $N_{B}$ is $B_{\text {attribute }}$, the correlation coefficient could be expressed by $R_{A B}$, then $R_{A B}=\left(A_{\text {attribute }} \cap\right.$ $\left.B_{\text {attribute }}\right) /\left(A^{\text {attribute }} \cup B_{\text {attribute }}\right)$. According to the definition, $R_{A B} \in[0,1]$, if the value of $R_{A B}$ is high, the attributes of $N_{A}$ and $N_{B}$ are similar.

Definition 2 Threshold: When a device wants to join into a group, it need calculate the correlative degree with the devices in the group. If all values are higher than a certain value, it will be allowed to enter the group, and this certain value is called Threshold(T). So, if the leader device $N_{A}$ creates a new group $\mathrm{G}$, and all the devices in the group $\mathrm{G}$ are denoted as $N_{G}$, that is $N_{G} \geqslant 1$. When a new device $N_{B}$ want to enter the group, if $N_{G}>1$, and $\left|N_{C}\right| R_{B C} \geq T, N_{C} \in N_{G} \mid \geq 2$, or $\left|N_{G}\right|=1$, and $R_{A B} \geq T$, the new device could enter this group.

If a device doesn't need the sharing resource again, and it wants to get a new kind of resource from another group, it could apply to exit current group. If the leader device needs to exit the group, the mobile agent of managing group must select a new leader for the group.

\subsection{Resource Management Technique}

After creating a group, the mobile agent needs to control the distribution of resource. To describe the resource management technique better, we put forward some definitions and theorems.

Definition 3 Atomic resource message: It is also called atomic message (AM) for short. It is the minimum block of message which could be cut into, but still could be used in the future. Only AM could be allowed to transfer in the group at MP2P network. We use the mark $R^{*}$ to denote it.

Definition 4 Combinative resource message: It is also called combinative message $(\mathrm{CM})$ for short. It makes up of a serial of AM. The method of combination concludes combination on different dimension, combination on the same dimension, and both of above. We use the mark $\mathrm{R}$ to denote it. Then $\mathrm{R}=\mathrm{n} R^{*}(n \geq 2)$.

Definition 5 Resource binary tree (RBT): It is used to record a certain resource of group. In binary tree, the node represents the handheld mobile device, and the branch represents the two devices with a direct communication link. The leaf of tree represents the AM of group at a certain device.

In RBT, there is a branch between two nodes. This represents they could share resource with greater efficiency and speed, and the nodes connected by left branch are better than the nodes connected by right branch. According to 
the definition, only the AM could be represented by RBT, but we usually need $\mathrm{CM}$ as we work. So we must ensure that they could transform into each other.

Theorem 1: In MP2P network, CM could be combined by AM.

Proof. Let A, B, C, D are AMs, A, B at the same dimension, C, D at the different dimension, and $\mathrm{A}, \mathrm{B}$ could not be combined with $\mathrm{C}$, D, Then $R_{A B}=R_{A}^{*}+R_{B}^{*}$, $R_{C D}=R_{C}^{*} \cup R_{D}^{*}$. So, $R_{A B C D}=R_{A B}+R_{C D}=\left(R_{A}^{*}+R_{B}^{*}\right)+\left(R_{C}^{*} \cup R_{D}^{*}\right)$ That is the AMs could transform into a CM.

Theorem 2: In MP2P network, AM could be transformed by CM and AMs.

Proof. Let A, B, C, D are AMs, $R_{A B C D}$ is a CM, which is combined by A, B, C, D. With theorem 1, we know: $R_{A B C D}=\left(R_{A}^{*}+R_{B}^{*}\right)+\left(R_{C}^{*} \cup R_{D}^{*}\right)$. If we want to get A or B, Then, $R_{A}^{*}=R_{A B C D}-R_{B}^{*}-\left(R_{C}^{*} \cup R_{D}^{*}\right)$ or $R_{B}^{*}=R_{A B C D}-R_{A}^{*}-\left(R_{C}^{*} \cup R_{D}^{*}\right)$. We obtain that $\mathrm{A}$ or $\mathrm{B}$ could be transformed. If we want to get $\mathrm{C}$ or $\mathrm{D}$, Then, $\left(R_{C}^{*} \cup R_{D}^{*}\right)=R_{A B C D}-R_{A}^{*}-R_{B}^{*}$. That is $R_{C}^{*}=\left(R_{A B C D}-R_{A}^{*}-R_{B}^{*}\right) / R_{D}^{*}$ or $R_{D}^{*}=\left(R_{A B C D}-R_{A}^{*}-R_{B}^{*}\right) / R_{C}^{*}$. We obtain that $\mathrm{C}$ or $\mathrm{D}$ could be transformed. So, AM could be transformed by a CM and the other AMs.

Using the RBT, according to theorem 1 and 2, we can buildup a route from the user to the resource or part of the resource meeting the request.

\subsection{Pipe Technique}

Definition 6 Pipe technique: In MP2P network, pipe technique is that a device could get a resource from another group directly.

In MP2P network, pipe technique is a core technique of mobile education field, and we will introduce it in next paper in detail.

\section{Conclusions}

In geology field course, the resources only are saved on the server, so anything must be got from it. In MP2P network, we suppose the server is a number of the group, and call it A0. A1, A2, A3, A4 all could get the resources from A0 through the index of RBT if they need. So, no matter which method is used to get the map, the fee is equal. However, after anyone of them gets the map from A0, the others don't need get the map from A0, and they could get it via the MP2P network, so the fee for communication is descended.

With MP2P, any group that requires a collaborative channel for achieving an educational goal could be brought to success with less time and money. So, the MP2P technique has a broad prospect at M-Education field.

\section{References}

1. Carroll, J.M. Rosson, M.B. Isenhour P.L. : Designing Our Town: MOOsburg, International Journal of Human-Computer Studies, Vol.54, No.5, pp. 725-751.

2. Liang, Y. Ci, L. Su, F. : Dynamic Location Service Based on Measurement of Moving Regularity in Mobile Computing Environment[C]. 6th International Symposium on Test and MeasurementVol. 8, Jul. 2005: pp.7987-7990. 